\title{
Eficacia del JAL 90458 sobre el recrecimiento de placa en pacientes con aparatología fija multibrackets
}

\section{Efficacy of JAL 90458 in the regrowth of plaque in patients with orthodontic braces}

\author{
Claudet Angulo PC*, Alió Sanz JJ**, Ramírez Mingorance EM***, \\ Iglesias Conde $\mathrm{C} * * * *$ Manso Platero FJ*****
}

\section{RESUMEN}

Introducción. El objetivo fue evaluar la eficacia del JAL 90458 sobre el recrecimiento de placa en pacientes con aparatología fija "multibrackets".

Material y métodos. Participaron 16 sujetos (11 mujeres y 5 hombres) entre 12 a 39 años de edad. El estudio fue realizado bajo condiciones de un ensayo clínico, aleatorizado, doble ciego y paralelo de grupos balanceados usando un diseño de recrecimiento de placa. Los sujetos recibieron una profilaxis oral y suspendieron toda medida de higiene oral por los siguientes 4 días, usando sólo el gel asignado. Se evaluó el Índice de placa de Quigley-Hein modificado por Turesky (IPQH-T), el Índice de placa bracket (IPb) y el Índice de Sangrado al Sondaje (ISS). Para el análisis de datos se utilizó el test de la $t$ de Student para muestras independientes, el test exacto de Fisher y el coeficiente de correlación de Pearson.

Resultados. Se obtuvieron diferencias estadísticamente significativa entre el JAL 90458 y el gel control en el IPQH-T (2,52 $\pm 0,57$ vs $1,78 \pm 0,79, p<0,05)$, así como en el IPb $(2,04 \pm 0,73$ vs $1,27 \pm 0,59, p<0,05)$, y ISS $(1,24 \pm 0,20$ vs $0,48 \pm 0,24, p<0,05)$. Hubo una alta correlación entre el IPb y el IPQH-T $(r=0,94, p<0,01)$. La ecuación de regresión lineal para predecir el valor del IPQH-T a través del IPb fue: IPQH-T=0,568+0,956 * IPb. Discusión. La aplicación del JAL 90458 en pacientes con aparatos fijos "multibrackets" demostró ser eficaz en la disminución del recrecimiento de placa bacteriana. No se detectó ningún efecto secundario clínico relevante. Palabras clave: Enjuagues bucales, triclosán, placa dental, brackets, ensayos clínicos, índice de placa.

\section{SUMMARY}

Introduction. The objective was test the efficacy of JAL 90458 on the regrowth of plaque in patients with orthodontic braces.

Material and methods. A total of 16 subjects ( 11 women and 5 men) between the ages of 12 and 39 took part in this study. The study was conducted under clinical trial conditions, double blind, parallel and randomized in balanced groups using a plaque regrowth design. The subjects had a professional teeth-cleaning and were

* Estudiante del Máster de Ortodoncia del Instituto Tecnológico Europeo de las Ciencias Odontológicas. Universidad de Alcalá. Alcalá de Henares. Madrid.

** Profesor titular de Ortodoncia. Departamento de Odontología. Universidad Complutense de Madrid.

*** Profesor del Instituto Tecnológico Europeo de las Ciencias Odontológicas. Universidad de Alcalá. Alcalá de Henares. Madrid.

**** Profesor de la Universidad de Alcalá. Alcalá de Henares. Madrid.

***** Profesor del Instituto Tecnológico Europeo de las Ciencias Odontológicas. Universidad de Alcalá. Alcalá de Henares. Madrid. 
instructed to not use any kind of oral hygiene measures for the next four days, using only the gel that had been assigned to them. We took into account the following parameters: the Quigley - Hein plaque index, modified by Turesky (QHPI-T), Plaque - Bracket Index (PIb) and the Bleeding on Probing Index (BOP). Analysis of the data was done using a Student's t-test, Fisher's exact test and Pearson's correlation coefficient.

Results. Statistically significant differences between the JAL 90458 group and the control gel group were obtained in the QHPI-T (2.52 \pm 0.57 vs $1.78 \pm 0.79, \mathrm{p}<0.05)$, as well as in the Plb $(2.04 \pm 0.73$ vs $1.27 \pm 0.59$, $\mathrm{p}<0.05)$, and BOP $(1.24 \pm 0.20$ vs $0.48 \pm 0.24, \mathrm{p}<0.05)$. There was a high correlation between the PIb and the QHPI-T $(r=0.87, p<0.01)$. The linear regression equation to predict the value of the QHPI-T using the PIb was: QHPI-T $=0.568+0.956 *$ PIb.

Discussion. The application of JAL 90458 in patients with orthodontic braces was shown to be effective in the diminishing the regrowth of bacterial plaque. We did not detect any clinically relevant side effect.

Key words: Mouthrinses, triclosan, dental plaque, orthodontic braces, clinical trials, plaque index.

Fecha de recepción: 5 de septiembre de 2012.

Aceptado para publicación: 12 de noviembre de 2012.

Claudet Angulo PC, Alió Sanz JJ, Ramírez Mingorance EM, Iglesias Conde C, Manso Platero FJ. Eficacia del JAL 90458 sobre el recrecimiento de placa en pacientes con aparatología fija multibrackets. Av. Odontoestomatol 2013; 29 (5): 245-253.

\section{INTRODUCCIÓN}

Los aparatos de ortodoncia fija crean áreas retentivas que impiden el adecuado control de placa bacteriana y permiten el aumento de desechos, placa y cálculo, que causan caries y enfermedad periodontal (1-8). Por estas razones, el control mecánico de la placa debería estar complementado por el control químico, el asesoramiento dietético y el cuidado profesional (4).

El valor potencial de los agentes antimicrobianos en el control de placa y gingivitis está bien establecido. Numerosos agentes antimicrobianos han sido evaluados y han demostrado diferentes grados de actividad antimicrobiana, $(9,10)$ así como la presencia de efectos secundarios por su uso prolongado (3).

El JAL 90458 (producto de investigación) es un nuevo producto que disminuye la fricción producida por el contacto entre el bracket y el arco, lo que permite un movimiento dental más rápido. A parte de su propiedad antifricción, este producto incorpora en su composición otras sustancias como el Triclosán, citrato de cinc y alantoína que pueden ser una alternativa que evite estos efectos $(11,12)$.
El Triclosán uno de los componentes del JAL 90458 tiene propiedades antiplaca, antigingivitis y anticálculo (13-16) carece de efectos secundarios tanto locales como sistémicos y no altera la ecología microbiana oral $(17,18)$. El Citrato de cinc potencia la eficacia clínica del JAL 90458 debido a que aumenta la sustantividad del triclosán $(13,18)$ y la alantoína es un regenerador de los tejidos gingivales.

La adecuada formulación de estos agentes activos en forma de dentífricos/colutorios u otras presentaciones tales como esprays, tiras, barnices y geles es extremadamente importante para mejorar su eficacia $(7,19,20)$. El JAL 90458 debido a su formulación en gel permite una mejor adhesión al diente y mucosa lo que mejoraría su eficacia.

La composición del JAL 90458 hace posible usarlo como un agente coadyuvante del control mecánico de la placa durante el tratamiento de ortodoncia fija.

El objetivo principal de este estudio fue evaluar la eficacia del JAL 90458 sobre el recrecimiento de placa en comparación con un gel control, debido a que hasta donde sabemos, no han sido descritos ensayos clínicos aleatorizados con triclosán en for- 
ma de gel en este grupo de población. Los objetivos secundarios fueron evaluar el efecto del JAL 90458 en la reducción del sangrado al sondaje, así como determinar si existe correlación entre índice de placa de Quigley-Hein modificado por Turesky (IPQH-T) (21) y el índice de placa bracket (IPb) (22). Si hubiera una alta correlación $(r>0,8)$ entre las variables, se establecería una ecuación de regresión lineal. Otro objetivo secundario fue evaluar la frecuencia de los efectos secundarios de ambos geles.

\section{MATERIAL Y MÉTODOS}

Un total de 16 sujetos (11 mujeres y 5 varones) que estaban bajo tratamiento ortodóncico en la División de Ortodoncia del Instituto Europeo de Postgrado (IEP), con un rango de edad entre 12-39 años participaron en este estudio después de firmar el consentimiento informado.

Todos los sujetos llevaban aparatos fijos "multibrackets" en incisivos, caninos, premolares y bandas en primeros molares prescripción Roth slot .018" o .022", por al menos 6 meses. Los sujetos tuvieron un buen estado de salud sin historia médica o farmacológica que pudiera comprometer los resultados del estudio, además de un buen estado de salud oral, con un mínimo de 20 dientes permanentes. Se excluyó a los sujetos con historia previa de alergias múltiples conocidas, embarazo o en periodo de lactancia.
Antes de empezar, el estudio fue aprobado por el Comité Ético de Investigación Clínica del Hospital Clínico de San Carlos (CEHCSC) en Madrid.

Fue un ensayo clínico doble ciego, aleatorizado, de grupos paralelos usando un diseño clásico de recrecimiento de placa de 4 días (23), como se ilustra en la figura 1. La exploración y selección de los voluntarios fue llevado a cabo por un solo investigador, éste lo registró en el Cuaderno de Recogida de Datos (CRD) quien también registró todas las visitas del ensayo.

Antes de empezar la fase experimental el investigador realizó una evaluación de la condición médica y bucal del sujeto. Si los sujetos reunían los criterios de inclusión/exclusión, el investigador les informaba los objetivos del estudio y les pidió su participación voluntaria. El investigador asignó a cada sujeto un número de código, el cual se dio de acuerdo al orden de llegada del sujeto y se le asignó a cada sujeto el primer número aleatorio libre de la lista de aleatorización.

En la visita 0 (día 15), a cada sujeto se le realizó un examen de tejidos duros y blandos. Una única higienista dental les realizó la profilaxis dental y les enseñó la Técnica de cepillado de Bass (24), recibieron además un kit de higiene oral que contenía un cepillo dental estándar (Vitis ${ }^{\circledR}$ Access Medium, DENTAID, Cerdanyola, España) y una pasta dental convencio-

\begin{tabular}{|c|c|c|c|c|}
\hline $\begin{array}{l}\text { Visita } 0 \\
\text { Evaluación y } \\
\text { selección }\end{array}$ & $\begin{array}{l}\text { Fase de higiene } \\
\text { (15 días) }\end{array}$ & Visita 1(dia 1) & \multirow[t]{2}{*}{$\begin{array}{c}\text { Día 2-4 } \\
3 \times \text { diario } \\
\text { con JAL } 90458 \\
3 \mathrm{ml} / 3 \mathrm{~min}\end{array}$} & Visita 2 (día 5) \\
\hline$\perp$ & & ـ & & $ل$ \\
\hline $\begin{array}{l}\text { Datos personales. } \\
\text { Historia médica. } \\
\text { Estado dental. } \\
\text { Limpieza dental } \\
\text { profesional. } \\
\text { Instrucciones de higiene } \\
\text { oral (cepillarse los dientes } \\
\text { con la técnica de Bass) }\end{array}$ & \multicolumn{3}{|c|}{$\begin{array}{c}\text { Estado dental. } \\
\text { ISS. } \\
\text { Limpieza dental profesional. } \\
\text { Indicaciones: } \\
\text { Abstenerse del cepillado } \\
\text { (4 dias) } \\
\text { Aplicación del gel asignado } \\
\text { (después de las comidas } \\
\text { principales) }\end{array}$} & $\begin{array}{c}\text { ISS } \\
\text { IQH-T } \\
\text { IPb } \\
\text { Seguridad, efectos } \\
\text { adversos. } \\
\text { Limpieza dental } \\
\text { professional. }\end{array}$ \\
\hline
\end{tabular}

Fig. 1. Esquema del ensayo clínico. 
nal (Colgate ${ }^{\circledR}$ protección caries, Colgate - Palmolive Co., New York, United States). Se les indicó cepillarse minuciosamente durante dos semanas antes de empezar la fase experimental y evitar el uso de cualquier enjuague bucal.

En la visita 1 (día 1), 15 días después de la visita 0 , a los sujetos se les realizó un examen oral para verificar el cumplimiento de los criterios de inclusión y se les registró el Î́ndice de Sangrado al Sondaje (ISS). Los sujetos recibieron una sesión de profilaxis oral y se les entregó el gel asignado. Los sujetos siguieron con su dieta normal pero el gel asignado fue el único procedimiento de higiene oral permitido durante todo el periodo experimental. Se les instruyó a los sujetos para que se apliquen el gel asignado en el margen gingival de la encía tanto en vestibular como en palatino/lingual, así como también en el cuerpo y alrededor del brackets, tres veces al día por cuatro días, de acuerdo al siguiente régimen diario: 3 ml por 3 minutos tras el desayuno, comida y cena. No se permitió ninguna comida inmediatamente después de la aplicación del gel (hasta 30 minutos).

En la visita 2 (día 5), después de la última aplicación del gel por la mañana, se llevó a cabo la visita final. Después de evaluar el ISS fue evaluado el IPQH-T e $\mathrm{IPb}$. Para evaluar la aparición de efectos secundarios, se les preguntó a los sujetos si ellos habían tenido hormigueo, alteración del gusto, boca seca y/ o sensación de quemazón. Al final del estudio, después del examen oral, los pacientes tuvieron una profilaxis dental.

Se usaron las siguientes formulaciones de investigación: Gel experimental antifricción (JAL 90458) el cual contiene triclosan $(0,30 \mathrm{~g})$, citrato de cinc $(0,05 \mathrm{~g})$, fluoruro de sodio $(0,124 \mathrm{p} / \mathrm{p})$, polisorbato 80 (n/e), alantoína (N/S), aloe barbadensis, flavor, olea European oil, water, CI 47005) y el gel control (Gel de Flúor Pierrot ${ }^{\circledR}$ ), el cual contiene monofluorfosfato de sodio $(0,04 \mathrm{p} / \mathrm{p})$ y fluoruro de sodio $(0,01$ $\mathrm{p} / \mathrm{p})$. Estos geles fueron entregados en jeringas idénticas codificadas. Los sujetos recibieron cuatro jeringas de gel (3 ml). Para verificar el grado de cooperación, cada paciente tenía que asistir a todas sus visitas y en la visita 3 debió de traer todas las jeringas usadas.
El índice de placa fue evaluado el día 5 de acuerdo al criterio del IPQH-T (21). Se registraron los 6 sitios alrededor de cada diente mesiobucal, mediobucal, distobucal, mesiolingual/palatal, mediolingual/palatal y distolingual/palatal. El IPb fue evaluado el día 5 sobre y alrededor de los brackets de acuerdo al criterio diseñado por Trimpeneers (22). La puntuación de la placa fue realizada después de la pigmentación con Floxina B al 4\% (Plac Control ${ }^{\circledR}$, DENTAID, Cerdanyola, España).

El ISS (22) fue evaluado los días 1 y 5 en los seis sitios alrededor de cada diente de acuerdo al criterio utilizado por Trimpeneers. Para el sondaje, se empleó una sonda periodontal UNC 15 (Hu-Friedy). Cuando la sonda no podía ser insertada a lo largo del eje axial del diente, no se puntuó. Esto principalmente ocurría en las superficies bucales donde los brackets interferían con el sondaje.

\section{Análisis estadístico}

Los análisis de datos se realizaron usando el SPSS versión 16. Los datos cumplieron los criterios de normalidad (25). Los valores medios de los parámetros clínicos fueron comparados entre los grupos de tratamiento mediante el test de la $t$ de Student para muestras independientes (26). El nivel de significación se fijó en $p=0,05$. Las comparaciones entre los grupos sobre los efectos secundarios se realizaron mediante la prueba exacta de Fisher (27). Se realizó la Correlación de Pearson entre IPQH-T y el IPb. La alta correlación $(r>0,8)$ encontrada entre estas variables permitió establecer la ecuación de regresión lineal (28).

\section{RESULTADOS}

El estudio fue iniciado en un principio con 18 pacientes. Durante la realización del estudio fueron excluidos dos pacientes, por no acudir a las visitas. De estos 16 pacientes, 5 fueron varones y 11, mujeres. No hubo diferencias significativas entre el JAL 90458 y el gel control en relación a las variables edad y sexo, como se ilustra en la tabla 1.

Todos los pacientes, tanto del grupo del JAL 90458 como del grupo control, iniciaron con un IPQH-T 


\begin{tabular}{|l|c|c|}
\hline \multicolumn{3}{|c|}{ TABLA 1.- NO EXISTEN DIFERENCIAS } \\
ENTRE HOMBRES Y MUJERES EN \\
RELACIÓN A LAS VARIABLES EDAD \\
Y SEXO (*) \\
\hline Datos demográficos & $\begin{array}{c}\text { Control } \\
\mathbf{n}=8\end{array}$ & $\begin{array}{c}\text { JAL } 90458 \\
\mathbf{n}=8\end{array}$ \\
\hline Edad & $18,25 \pm 9,19^{*}$ & $21,18 \pm 7,93^{*}$ \\
\hline $\begin{array}{l}\text { Sexo: } \\
\text { - Mujeres } \\
\text { - Varones }\end{array}$ & 4 & $7^{*}$ \\
\hline
\end{tabular}

Test de la t de Student $(p=0,11)$ y prueba exacta de Fisher $(p=0,28)$.

igual a 0 antes de que les fuera asignado el gel. Ambos grupos aumentaron los niveles de placa durante el periodo experimental. El aumento del IPQH$\mathrm{T}$ en el grupo experimental fue menor que el grupo control. Se encontró diferencias estadísticamente significativas a favor del JAL 90458 (*p<0,05), como se detalla en la figura 2 .

La mayor puntuación del IPB lo obtuvo el grupo control. Estas diferencias observadas entre ambos grupos fueron estadísticamente significativas $(p<0,05)$, como se ilustra en la figura 3.

Se encontró una alta correlación entre el IPQH-T y el $\mathrm{IPb}(\mathrm{r}=0,94, \mathrm{P}<0,01)$. Esto nos permitió establecer la ecuación de regresión lineal para predecir el valor del IPQH-T a través del IPb, este fue: IPQH-T =0,568 $+0,956 * \mathrm{IPb}$, como se detalla en la figura 4 .

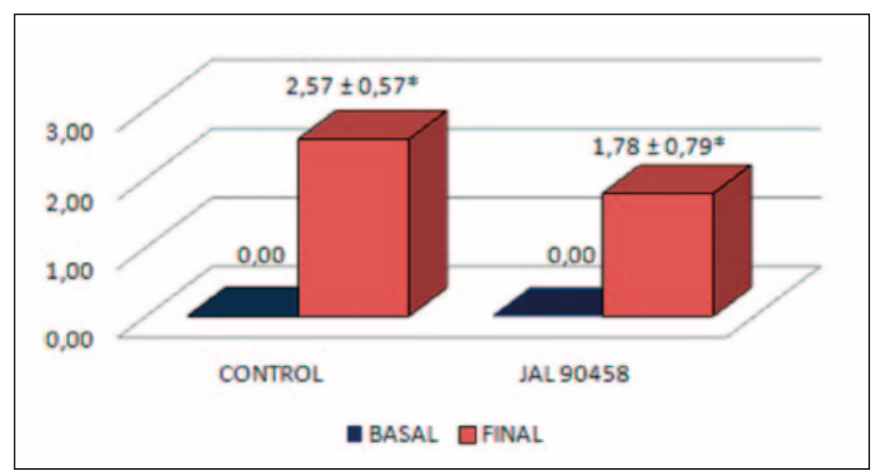

Fig. 2. Índice de placa de Quigley-Hein modificado por Turesky (IPQH-T)

* Estadísticamente significativo (test de la $t$ deStudent, $\mathrm{p}<0,05$ ).

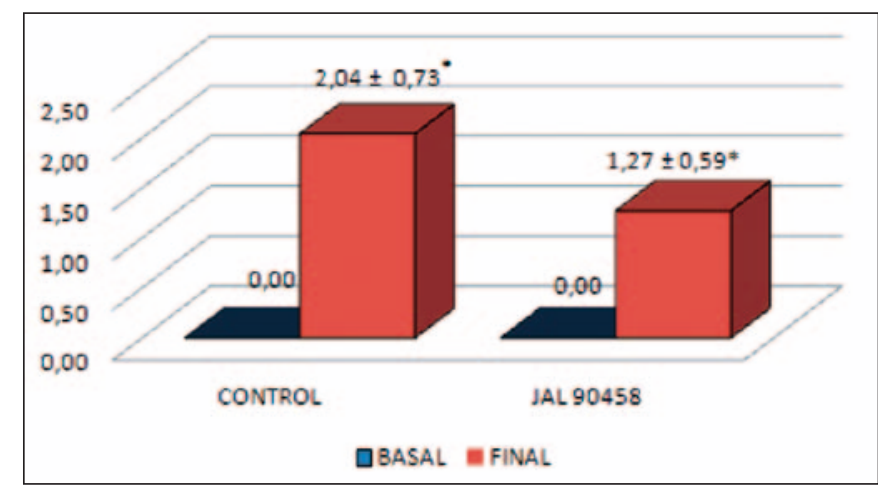

Fig. 3. Índice de placa bracket (IPb).

* Estadísticamente significativo (test de la $t$ de Student, $\mathrm{p}<0,05$ ).

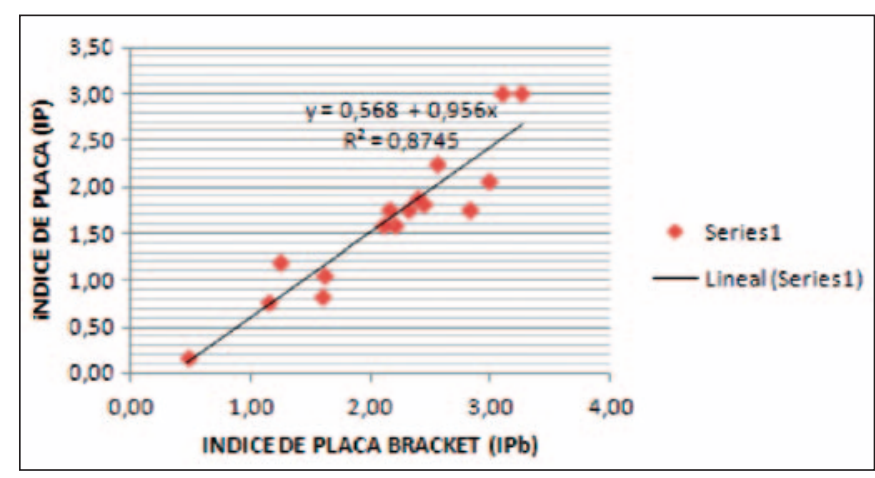

Fig. 4. Gráfico de dispersión y determinación de la ecuación de regresión lineal.

La condición gingival se valoró a través del ISS. La menor puntuación del ISS lo obtuvo el grupo experimental. Estas diferencias encontradas fueron estadísticamente significativas y favorables al JAL 90458, como se detalla en la figura 5.

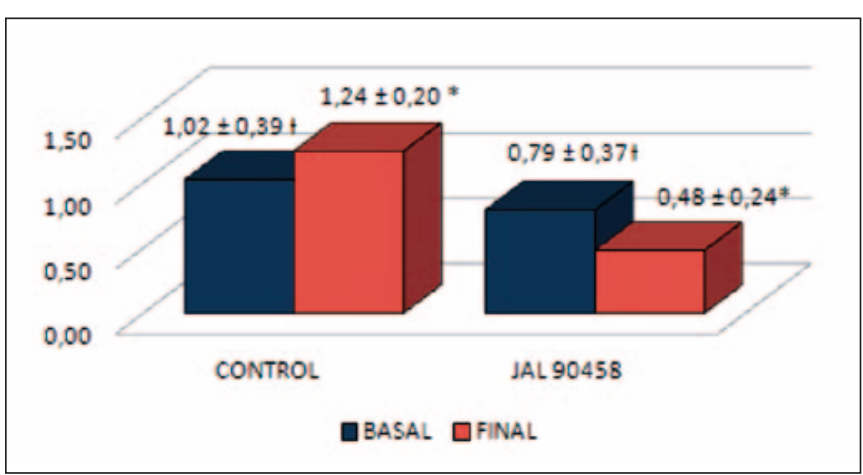

Fig. 5. Índice de sangrado al sondaje (ISS).

$\dagger$ No significativo (test de la $t$ de Student).

* Estadísticamente significativo (test de la $t$ de Student, $\mathrm{p}<0,001$ ). 
La sensación de boca seca (xerostomía) fue el efecto secundario descrito con más frecuencia, pero estas diferencias no fueron estadísticamente significativas entre los grupos, como se ilustra en la tabla 2.

\section{DISCUSIÓN}

La acumulación de placa en pacientes con tratamiento ortodóncico es común debido a la dificultad de controlar la higiene oral por la presencia de bandas, arcos, "brackets" y ligaduras dentro de la boca (29) Por este motivo, el control mecánico de la placa, debe ser complementado con el control químico, control profesional y el asesoramiento dietético $(4,13)$.

Los resultados del presente trabajo demostraron que el JAL 90458 fue significativamente superior al gel control en el control del recrecimiento de placa. No hay estudios con que compararlo.

Numerosos estudios sobre recrecimiento de placa han evaluado la eficacia de diferentes colutorios tales como clorhexidina, $(30,31)$ aceites esenciales (10), hidrocloruro de benzamida-cetilpiridinio, (32) cetilpiridinio, $(9,32)$ fluoruro de estaño, (9) polihexametileno biguanina (33) y el agente bisfenólico triclosán (10, 30, 34). La actividad de clorhexidina según estos estudios es superior a otros agentes pero sus efectos secundarios tales como tinción dental o disgeusia, han animado el desarrollo o uso de agentes alternativos $(13,16)$. El triclosán, unido a otras sustancias como PVM/AM o citrato de cinc, aumentan su sustantividad lo que ha demostrado ser eficaz en la inhibición de la neoformación de placa bacteriana $(13,35)$.

Existen otros estudios a medio y largo plazo en pacientes con y sin tratamiento ortodónticos $(3,7,29)$ que analizan diferentes agentes antisépticos en diferentes formulaciones tales como colutorios (29) y pastas dentífricas $(13,36)$ como coadyuvantes de los procedimientos mecánicos de higiene oral. En tales estudios se demostró una reducción significativa de los parámetros clínicos periodontales.

El IPb descrito por Trimpeneers (22) fue utilizado para valorar la eficacia antiplaca de JAL 90458 en pacientes con tratamiento ortodóncico. Hasta donde sabemos no han sido descritos estudios sobre recrecimiento de placa que empleen este índice $\mathrm{y}$, por lo tanto, no tenemos con que compararlo.

Para determinar si existe una asociación lineal entre las variables IPQH-T y el IPb, utilizamos la prueba del Coeficiente de Correlación de Pearson. Se obtuvo un coeficiente de correlación muy alto. Por tal motivo,

\begin{tabular}{|c|c|c|c|c|c|c|c|c|}
\hline \multicolumn{9}{|c|}{ TABLA 2.- EFECTOS ADVERSOS DESPUÉS DEL TRATAMIENTO } \\
\hline & \multicolumn{2}{|c|}{ Hormigueo } & \multicolumn{2}{|c|}{ Cambio de gusto } & \multicolumn{2}{|c|}{ Boca seca } & \multicolumn{2}{|c|}{$\begin{array}{c}\text { Sensación } \\
\text { de quemazón }\end{array}$} \\
\hline & $\mathrm{n}^{\circ}$ & $\%$ & $\mathbf{n}^{\circ}$ & $\%$ & $\mathbf{n}^{\circ}$ & $\%$ & $\mathrm{n}^{\circ}$ & $\%$ \\
\hline $\begin{array}{l}\text { Control } \\
\text { - Ausente } \\
\text { - Suave } \\
\text { - Moderado } \\
\text { - Severo }\end{array}$ & $\begin{array}{l}7 \\
1 \\
0 \\
0\end{array}$ & $\begin{array}{r}87,5 \\
12,5 \\
0,0 \\
0,0\end{array}$ & $\begin{array}{l}7 \\
1 \\
0 \\
0\end{array}$ & $\begin{array}{r}87,5 \\
12,5 \\
0,0 \\
0,0\end{array}$ & $\begin{array}{l}5 \\
2 \\
1 \\
0\end{array}$ & $\begin{array}{r}62,5 \\
25,0 \\
12,5 \\
0,0\end{array}$ & $\begin{array}{l}8 \\
0 \\
0 \\
0\end{array}$ & $\begin{array}{r}100,0 \\
0,0 \\
0,0 \\
0,0\end{array}$ \\
\hline $\begin{array}{l}\text { JAL } 90458 \\
\text { - Ausente } \\
\text { - Suave } \\
\text { - Moderado } \\
\text { - Severo }\end{array}$ & $\begin{array}{l}8 \\
0 \\
0 \\
0\end{array}$ & $\begin{array}{r}100,0 \\
0,0 \\
0,0 \\
0,0\end{array}$ & $\begin{array}{l}6 \\
1 \\
0 \\
1\end{array}$ & $\begin{array}{r}75 \\
12,5 \\
0,0 \\
12,5\end{array}$ & $\begin{array}{l}4 \\
3 \\
1 \\
0\end{array}$ & $\begin{array}{r}50,0 \\
37,5 \\
12,5 \\
0,0\end{array}$ & $\begin{array}{l}7 \\
0 \\
0 \\
1\end{array}$ & $\begin{array}{r}87,5 \\
0,0 \\
0,0 \\
12,5\end{array}$ \\
\hline
\end{tabular}

No significativo (test chi al cuadrado, ${ }^{*} p<0,01$ ). 
fue generada la ecuación de regresión lineal para predecir el IPQH-T a través del IPb que podría ser de utilidad.

La placa supragingival es un factor de riesgo para el desarrollo de la gingivitis. Al ser nuestro estudio sobre recrecimiento de placa; no se puede predecir la acción directa de estos agentes sobre los tejidos gingivales; pero el estado de salud gingival fue evaluado a través del ISS. Los resultados del presente estudio demostraron que el JAL 90458 fue significativamente superior al gel control en la reducción del ISS; esto fue debido a sus propiedades antiinflamatorias (36), además de antimicrobianas $(13,14)$.

Los efectos secundarios descritos en este estudio fueron hormigueo, alteración del gusto, sensación de quemazón y boca seca. No se detectó ningún efecto secundario clínico relevante La boca seca fue el efecto secundario más frecuente sin diferencias estadísticamente significativas entre ambos grupos.

No disponemos de estudios previos acerca de los posibles efectos adversos a largo plazo de los componentes del JAL 90458 en pacientes bajo tratamiento ortodóncico; sin embargo, el triclosán es uno de los agentes quimioterapéuticos más seguros, tolerables y eficaces de los que disponemos en la actualidad $(30,34,36)$.

Se recomienda la realización de ensayos clínicos aleatorizados (ECA) a largo plazo que evalúen la eficacia de la aplicación del JAL 90458 sobre los parámetros clínicos y microbiológicos en pacientes con aparatología fija de ortodoncia de acuerdo a las recomendaciones de la Asociación Dental Americana (ADA). Además se pueden realizar otros estudios que evalúen los efectos cuantitativos y cualitativos de la aplicación del JAL 90458 sobre los cambios microbiológicos de la microflora subgingival en adolescentes con aparatos fijos multibrackets.

En conclusión, la aplicación del JAL 90458 demostró ser más eficaz sobre el recrecimiento de placa que el gel control en pacientes con aparatología fija "multibrackets".

Hubo una reducción significativa en el sangrado al sondaje a favor del JAL 90458. Además se encon- tró una alta correlación entre el IPQH-T y el IPb. La ecuación de regresión estimada para predecir el valor del IPQH-T a través del IPb fue: IPQH-T = 0,568 $+0,956 * \mathrm{IPb}$.

Se encontró una baja frecuencia de efectos secundarios. La xerostomía fue el efecto secundario más común en ambos grupos sin presentar diferencias estadísticamente significativas. Es necesario realizar más ensayos clínicos para confirmar estos resultados.

\section{BIBLIOGRAFÍA}

1. Anderson GB, Bowden J, Morrison EC, Caffesse RG. Clinical effects of chlorhexidine mouthwashes on patients undergoing orthodontic treatment. Am J Orthod Dentofac Orthop 1997 Jun; 111(6): 606-12.

2. Attack NE, Sandy JR, Addy M. Periodontal and Microbiological Changes Associated with the placement of orthontic appliances. A review. J Periodontol 1996 Feb;67(2):78-85.

3. Brightman LJ, Terezhalmy GT, Greenwell H, Jacobs M, Enlow DH. The effects of a $0.12 \%$ chlorhexidine gluconate mouthrinse on orthodontic patients aged 11 through 17 with established gingival. Am J Orthod Dentofac Orthop 1991 Oct;100(4): 324-9.

4. Gehlen I, Netuschil L, Berg R, Reich E, Katsaros C. The Influence of a $2 \%$ Chlorhexidine Mouthrinse on Plaque Regrowth in Orthodontic Patients. A randomized prospective study. Part I: clinical parameters. J Orofac Orthop 200;61(1): 54-62.

5. Hägg U, Kaveewatcharanont P, Samaranayake YH. Samaranayake LP. The effect of fixed orthodontic appliances on the oral carriage of Candida species and Enterobacteriaceae. Eur J Orthod; 2004 Dec:26(6):623-9.

6. Ristic M, Vlahovic Svabic M, Sasic M, Zelic O. Clinical and microbiological effects of fixed orthodontic appliances on periodontal tissues in 
adolescents. Orthod Craneofacial Res 2007 Nov; 10(4):187-95.

7. Olympio KPK, Bardal PAP, de M Bastos JR, Buzalaf $M A$. Effectiveness of a chlorhexidine dentifrice in orthodontic patients: a randomized - controlled trial. J Clin Periodontol 2006 Jun;33(6):421-6.

8. Zachrisson S, Zachrsisson BU. Gingival condition associated with orthodontic treatment. Angle Orthod 1972 Jan;42(1):26-34.

9. Addy M, Greenman J Renton- Harper P, Newcombe R, Doherty F. Studies on stannous fluoride toothpaste and gel. J Clin Periodontol 1997 Feb; 24(2):86-91.

10. Moran J, Addy M, Newcombe R. A 4 - day plaque regrowth study comparing an essential oil mouthrinse with a triclosan mouthrinse. J Clin Periodontol 1997 Set;24(9 Pt 1):636-39.

11. Machuca G, Valencia S, Lacalle JR, Bullon P A clinical assessment of the effectiveness of a mouthwash based on triclosan an on Zea mays uses as supplements to brushing. Quintessence Int 1997 May;28(5):329-35.

12. Morrow Dan, Wood DP, Speechley M. Clinical effect of Subgingival chlorhexidine irrigation on gingivitis in adolescent orthodontic patients. Am J Orthod Dentofac Orthop 1992 May;101(5): 408-13.

13. Brading MG, Cromwell VJ, Green AK, DeBrabander S, Beasley T, Marsh PD. The role of triclosan in dentifrice formulations, with particular reference to a new $0.3 \%$ Triclosan Calcium carbonate - based system. In Dent J 2004; 54(5 Suppl 1):291-8.

14. Furuichi Y, Ramberg P, Krok L, Lindhe J. Short term effects of triclosan on healing following subgingival scaling. J Clin Periodontol 1997 Oct; 24 (10):777-82.

15. Rosling B, Wannfors B, Volpe AR, Furuichi Y, Ramberg P, Lindhe J. The use of a triclosan/ copolymer dentifrice may retard the progression of periodontitis. J Clin Periodontol 1997 Dec;24 (12):873-80.

16. Stephen KW, Saxton CA, Jones CL, Ritchie JA, Morrison T. Control of gingivitis and calculus by Dentifrice Containing a Zinc Salt and Triclosan. J Periodontol 1990 Nov;61(11):674-9.

17. Ciancio SG. Agents for the Management of Plaque and Gingivitis. J Dent Res 1992 Jul;71(7): 1450-4. Review.

18. Triratana T, Rustogi KN, Volpe AR, DeVizio W, Petrone M, Giniger M.. Clinical Effect of a new liquid dentifrice containing triclosan/copolymer on existing plaque and gingivitis. J Am Dent Assoc 2002 Feb;133(2):219-25.

19. Greenman J, Nelson JG. Hydrolysis of triclosan monophosphate by dental plaque and selected species of oral micro-organisms. J Dent Res 1996 Aug; 75(8):1578-84.

20. Gunsolley JC. Un metaanálisis de estudios de agentes antiplaca y antigingivitis a seis meses. $\mathrm{J}$ Am Dent Assoc 2007 Abril;2(2):77-85.

21. Turesky S, Gilmore N, Glickman I. Reduce Plaque Formation by the Chloromethyl Analogue of Victamine C. J Periodontol 1970 Abril; 41(1):41-3.

22. Trimpeneers LM, Wijgaerts IA, Grognard NA, Dermaut LR, Adriaens PA. Effect of electric toothbrushes versus manual toothbrushes on removal of plaque and periodontal status during orthodontic treatment. Am J Orthod Dentofacial Orthop 1997 May;111(5):492-7.

23. Addy M, Willis L, Moran J. Effect of toothpaste rinses compared with chlorhexidine on plaque formation during a 4-day period. J Clin Periodontol 1983 Jan;10(1):89-99.

24. Bass CC. An effective method of personal oral hygiene; part. II. J La State Med Soc 1954 Mar; 106(3):100-12.

25. Siegel S. The One Sample Case. The KolmogorovSmirnov One Sample Test. In: Siegel S. Non 
Parametric Statistics for the Behavior Science. The Graw-Hill Book Co: New York, United States; 1956.

26. Chilton NW. Comparison of means of two samples. In: Chilton NW, Design and Analysis in Dental and Oral Research. Praeger Publishers Division: New York; 1982.

27. Siegel S. The case of two independent samples. The Fisher Exact Probability Test. In: Siegel S. Non Parametric Statistics for the Behavior Science. The Graw-Hill BooK Co: New York; 1956.

28. Chilton NW. Regression and Correlation. In: Chilton NW. Desingn and Analysis in Dental and Oral Research. Praeger Publishers Division: New York; 1982.

29. Tufecki E, Casagrande ZA, Lindauer SJ, Fowler CE, Williams KT. Effectiveness of an Essential Oil Mouthrinse in Improving Oral Health in Orthodontic Pacients. Angle Orthod 2008 Mar; 78(2):294-8.

30. Moran J, Addy M, Roberts S. A comparison of natural product, triclosan and chlorhexidine mouthrinses on 4 - day plaque regrowth. J Clin Periodontol 1992 Sep;19(8):578-82.

31. Jenkins S, Addy $M$ y Newcombe RG. Dose response of chlorhexidine against plaque and comparison with triclosan. J Clin Periodontol 1994 Apr;21(4):250-5.

32. Herrera D, Santos S, Ferrú J, Barbieri G, Trombelli L, Sanz M. Efficacy of a $0.15 \%$ benzydamine hydrochloride and $0.05 \%$ cetylpyridinium chloride mouth rinse on 4 day de novo plaque formation. J Clin Periodontol 2005 Jun;32(6):595-603.

33. Arweiler NB, Henning G, Reich E, Netuschil L. Effect of an amine-fluoride-triclosán mouthrinse on plaque regrowth and biofilm vitality. J Clin Periodontol 2002 Apr;29(4):358-63.

34. Addy M. Chlorhexidine compared with other locally delivered antimicrobials. A short review. J Clin Periodontol 1986 Nov;13(10):957-64.

35. Barnes VM, Ritcher R, Vandeven M, Xu T, DeVizio W. Clinical Investigation of Antiplaque efficacy of a New variant of a commercially available triclosan-copolymer-fluoride dentifrice. J Clin Dent 2008;19(3):81-4.

36. Rosin M, Welk A, Kocher T, Majic-Todt A, Kramer A, Pitten FA. The effect of a polyhexamethylene biguanide mouthrinse compared to an essential oil rinse and a clhorhexidine rinse on bacterial counts and 4-day plaque regrowth. J Clin Periodontol 2002 May;29(5):392-9.

\section{CORRESPONDENCIA}

Dra. Paola Consuelo Claudet Angulo Máster de Ortodoncia del Instituto Tecnológico Europeo de las Ciencias Odontológicas Universidad de Alcalá de Hernares.

Madrid

Eugenio Salazar, 5, 2A

28002 Madrid

E-mail: pcca76@hotmail.com 